\title{
Marginal Folate Deficiency as a Possible Cause of Hyperhomocysteinaemia in Stroke Patients
}

\author{
Björn Hultberg', Anders Andersson ${ }^{1}$ and Arne Lindgren ${ }^{2}$ \\ 1 Department of Clinical Chemistry, University Hospital, Lund, Sweden \\ 2 Department of Neurology, University Hospital, Lund, Sweden
}

Summary: It has been reported that patients with vascular disease seem to increase their concentration of plasma homocysteine after the acute episode, whereas reexamined control subjects do not change their concentration of plasma homocysteine over time. Since the main determinants of plasma homocysteine are serum cobalamin, blood folate and serum creatinine we measured these quantities in 20 control subjects and 49 stroke patients in the acute phase and at reexamination 1.5-2 years after acute stroke onset. There were no significant differences between the levels of blood folate, serum cobalamin and serum creatinine in the acute and convalescent phase of all 49 stroke patients. However, we noted a significant decrease of blood folate concentrations in a subgroup of patients $(n=25)$ who had increased plasma homocysteine concentrations. Thus the increase in plasma homocysteine concentrations in this group of patients may partly be caused by a marginal folate deficiency.

\section{Introduction}

Greatly elevated plasma levels of homocysteine, a sulphydryl amino acid, are found in subjects with homocystinuria (1). These patients exhibit early arteriosclerosis, and arterial and venous thrombosis. Numerous studies have indicated that milder hyperhomocysteinaemia is also associated with increased risk of occlusive vascular diseases (2-7). Homocysteine is the demethylated derivative of methionine (1), and can be metabolized by two pathways, either catabolized by the transulphuration pathway to cysteine or remethylated to methionine, mainly by the folate and vitamin $\mathrm{B}_{12}$ dependent enzyme methionine synthase. Homocysteine accumulates when methionine synthase is impaired. Plasma levels of homocysteine have also been shown to be elevated in patients with cobalamin deficiency and folate deficiency $(8-14)$. Genetic factors such as heterozygosity for homocystinuria or the thermolabile variant of methylenetetrahydrofolate reductase seem to partly explain the aetiology of hyperhomocysteinaemia. Also a possible relation between diet, hyperhomocysteinaemia and arteriosclerosis has recently been suggested (15). However, little is known about the extent to which a marginal vitamin deficiency contributes to mild hyperhomocysteinaemia and vascular disease.

In an earlier study (16) we showed that the concentration of plasma homocysteine is significantly increased in stroke patients 1-2 years after the acute stroke onset, whereas reexamined control subjects showed no change in their concentration of plasma homocysteine over a similar period of time. Similar findings of increased plasma homocysteine in the post-acute phase of myocar- dial infarction $(17,18)$ have been reported. We also observed that plasma homocysteine concentrations do not change in the convalescence phase in patients (4) or over time in control subjects $(19)$. In earlier studies $(19,20)$ we found that the main determinants of plasma homocysteine in healthy subjects are age, serum cobalamin, blood folate and serum creatinine. In the present study we therefore measured the concentrations of serum cobalamin, blood folate and serum creatinine in 20 control subjects and 49 stroke patients in the acute phase and at reexamination $1.5-2$ years after acute stroke onset.

\section{Materials and Methods}

Study population

In the present study we reexamined 49 patients and 20 control subjects from earlier study populations $(16,20) 1.5-2$ years after the first examination. The patients were randomly selected from a consecutive series of 172 patients with stroke (16). The patient group consisted of 44 patients with cerebral infarction and 5 patients with intracerebral haemorrhage $(31$ males and 18 females aged $66 \pm 10$ years). Likewise, the control subjects were randomly selected from the 244 control subjects described earlier (20), who had been randomly selected from the local population records. The control group consisted of 14 males and 6 females aged $66 \pm 8$ years. We also analysed the concentration of plasma homocysteine, blood folate, serum cobalamin and serum creatinine in 41 males and 41 females (aged $65 \pm 16$ years) randomly selected from the larger control population (20).

\section{Assays}

Blood samples for determination of total plasma homocysteine were collected in evacuated tubes containing EDTA at about $8 \mathrm{a} . \mathrm{m}$. after an overnight fast and centrifuged within 15 minutes at $3000 \mathrm{~g}$ for 5 minutes. Both patients and control subjects were allowed at least 10 minutes of recumbent rest before blood sampling on the first occasion, whereas patients and control subjects at reinvestiga- 
tion were allowed $10-15$ minutes rest in a sitting position before venipuncture. The plasma was stored at $-70^{\circ} \mathrm{C}$ until analysis. Total plasma homocysteine (the sum of all homocysteinyl moieties, whether in sulphydryl or disulphide forms, free or protein-bound) was measured as previously described (21) by HPLC after reduction of disulphide bonds with dithiothreitol and deproteinization with sulphosalicylic acid. The method has a coefficient of variation of $4 \%$, calculated at the level of $15 \mu \mathrm{mol} / \mathrm{l}$.

Serum cobalamin and blood folate levels were determined by radioassay, using purified intrinsic factor and purified folate binding protein, at the Department of Clinical Chemistry, University Hospital, Lund (Vitamin $\mathrm{B}_{12} /$ Folate Dual RIA kit, Amersham, UK). The reference interval for serum cobalamin is $110-650 \mathrm{pmol} / 1$ and for blood folate $125-500 \mathrm{nmol} / \mathrm{l}$. Serum creatinine (upper reference limit $120 \mu \mathrm{mol} / \mathrm{l}$ ) was assayed with routine methods at the Department of Clinical Chemistry.

\section{Statistics}

The results are presented as means and SD. All statistical tests were two-tailed and the $5 \%$ level of significance was used to evaluate the results. Mann-Whitney $U$ test was used in the case of two independent samples and Spearman's rank correlation coefficients were calculated to test for monovariate relationship between different variables.

\section{Results}

The initial/reinvestigated mean values and SD of the concentrations of plasma homocysteine, blood folate, serum cobalamin and serum creatinine are given in table 1. The concentration of plasma homocysteine in control subjects did not change between the acute and convalescent phase, whereas that in patients increased significantly. The patients were divided into two groups (tab. 1). One group consisted of patients whose plasma homocysteine had increased by more than $2 \mu \mathrm{mol} / 1$ between the two analyses. This group showed a significantly decreased concentration of blood folate at reinvestigation. The other group of patients had similar plasma homo- cysteine concentrations $( \pm 2.0 \mu \mathrm{mol} / \mathrm{l})$ on both occasions. In this group there were no significant changes between the initial values of blood folate and the values at reinvestigation. Compared with the large group of healthy control subjects there were no significant deviations in the concentrations of blood folate, serum cobalamins or serum creatinine in any of the investigated groups (tab. 1).

Correlations between the initial values and the values at reinvestigation of each quantity in all patients were also analysed. Plasma homocysteine $\rho=0.66, p<0.001$ ), serum creatinine $(\rho=0.65, p<0.001)$ and blood folate $(\rho=0.61, p<0.001)$ correlated highly significantly between the two analyses, whereas serum cobalamin $(\rho=0.31, p<0.05)$ exhibited a lower degree of correlation.

The initial concentration of plasma homocysteine in all patients correlated significantly with the corresponding concentrations of serum creatinine $(\rho=0.46, p<0.01)$ and serum cobalamins $(\rho=-0.33, p<0.05)$, whereas there was no significant relation between the concentrations of plasma homocysteine and blood folate $(\rho=-0.17, p>0.05)$. The correlation between plasma homocysteine concentrations and age was significant $(\rho=0.27, p<0.05)$. At reinvestigation the concentration of plasma homocysteine in all patients correlated significantly with the corresponding concentrations of serum creatinine $(\rho=0.40, p<0.01)$ and blood folate ( $\rho=-0.40, p<0.01)$, whereas there was no significant relation between plasma homocysteine and serum cobal$\operatorname{amin}(\rho=-0.21, p>0.05)$. The correlation between plasma homocysteine concentrations and age was significant $(\rho=0.43, p<0.01)$.

Tab. 1 The concentrations of plasma homocysteine, blood folate, serum cobalamin and serum creatinine in different groups of control subjects and patients. Mean and SD are given.

\begin{tabular}{|c|c|c|c|c|}
\hline & $\begin{array}{l}\text { Plasma } \\
\text { homocysteine } \\
(\mu \mathrm{mol} / 1)\end{array}$ & $\begin{array}{l}\text { Blood } \\
\text { folate } \\
(\mathrm{nmol} / 1)\end{array}$ & $\begin{array}{l}\text { Serum } \\
\text { cobalamin } \\
(\mathrm{pmol} / \mathrm{l})\end{array}$ & $\begin{array}{l}\text { Serum } \\
\text { creatinine } \\
(\mu \mathrm{mol} / 1)\end{array}$ \\
\hline Control subjects $(\mathrm{n}=82)$ & $13.8 \pm 4.6$ & $356 \pm 178$ & $254 \pm 109$ & $86 \pm 24$ \\
\hline \multicolumn{5}{|l|}{ Control subjects $(\mathrm{n}=20)$} \\
\hline Initial values & $14.0 \pm 4.5$ & $400 \pm 211$ & $290 \pm 101$ & $90 \pm 16$ \\
\hline Reinvestigation & $12.6 \pm 3.8$ & $348 \pm 168$ & $280 \pm 101$ & $84 \pm 13$ \\
\hline \multicolumn{5}{|l|}{ All patients $(\mathrm{n}=49)$} \\
\hline Initial values & $12.6 \pm 4.3$ & $366 \pm 154$ & $245 \pm 72$ & $79 \pm 19$ \\
\hline Reinvestigation & $15.4 \pm 4.8^{* *}$ & $327 \pm 136$ & $299 \pm 136$ & $83 \pm 16$ \\
\hline \multicolumn{5}{|c|}{ Patients with increased plasma homocysteine $>2.0 \mu \mathrm{mol} / 1(\mathrm{n}=25)$} \\
\hline Initial values & $11.5 \pm 2.9$ & $399 \pm 191$ & $245 \pm 75$ & $78 \pm 20$ \\
\hline Reinvestigation & $17.5 \pm 4.3^{* * *}$ & $317 \pm 152^{*}$ & $274 \pm 114$ & $84 \pm 18$ \\
\hline \multicolumn{5}{|c|}{ Patients with unchanged values $\pm 2.0 \mu \mathrm{mol} / \mathrm{l}(\mathrm{n}=18)$} \\
\hline Initial values & $12.4 \pm 4.1$ & $329 \pm 94$ & $256 \pm 60$ & $77 \pm 19$ \\
\hline Reinvestigation & $13.0 \pm 4.2$ & $329 \pm 104$ & $317 \pm 141$ & $81 \pm 16$ \\
\hline
\end{tabular}

$* \mathrm{p}<0.05 ; \quad * * \mathrm{p}<0.01 ; \quad * * * \mathrm{p}<0.001$ compared to initial values. 


\section{Discussion}

The increase of plasma homocysteine in the convalescent phase after an acute vascular event, as reported earlier $(16,17,18)$ and as observed in the present study, might depend on lowered values at the time of the acute vascular event and/or an increase of plasma homocysteine levels in the convalescent phase. It is known that the acute inflammatory episode includes a number of systemic changes, e.g. dramatic increases of hepatic acute phase response plasma proteins and decreased synthesis of albumin. Since the major part of plasma homocysteine is bound to albumin, the binding of plasma homocysteine to protein may be decreased at the time of the acute vascular event. This leads to a higher renal clearance of homocysteine and possibly also to increased metabolic (hepatic) bio-availability, thereby eliminating homocysteine from plasma. That plasma homocysteine is lowered in the acute vascular event of a myocardial infarction is supported by the findings of Egerton et al. (18). These authors reported an approximate $25 \%$ decrease in plasma homocysteine during the acute phase response of the plasma proteins, compared with plasma homocysteine after the acute phase response had dissipated ( $>7$ days). In the present study there was no significant correlation between blood folate and plasma homocysteine at the time of the acute vascular episode, which might therefore be explained by the temporary lowering of the plasma homocysteine concentrations during this episode.

Conceivably, the increased plasma homocysteine level in the convalescent phase could be due to a real increase after the vascular event. The reason for such an increase is not obvious. In our earlier study (16) of 17 stroke patients there were no significant differences between the values for blood folate, serum cobalamin and serum creatinine in the acute and convalescent phases. We obtained similar results in the present study of all 49 stroke patients. However, we noted a significant decrease of blood folate concentrations in the subgroup of patients $(n=25)$ who increased their plasma homocysteine concentrations. Thus the increase in plasma homocysteine concentrations in this group of patients may partly be caused by the decrease of blood folate concentrations, since there is an intimate relationship between plasma homocysteine concentrations and folate status $(8-14)$. That plasma homocysteine concentrations are affected by folate status in the convalescence phase is also supported by the correlation between plasma homocysteine and blood folate at reinvestigation. The reason for decreased blood folate concentrations at reinvestigation is not obvious but it is known that several types of chronic inflammatory disorders lower blood folates (22). It is possible that the arteriosclerotic process in stroke patients has a similar influence on blood folates. Another possibility might be a change of lifestyle (less physical activity and/or poorer nutrition) after the stroke event, with a decreased folate intake.

Numerous studies have indicated that elevated plasma homocysteine concentrations are associated with increased risk of premature occlusive vascular diseases $(2-7)$. Genetic factors such as heterozygosity for homocystinuria or the thermolabile variant of methylenetetrahydrofolate reductase seem to largely account for the aetiology of hyperhomocysteinaemia. A possible relation between diet, hyperhomocysteinaemia and arteriosclerosis has recently been suggested (15). However, little is known about the extent to which a marginal vitamin deficiency contributes to mild hyperhomocysteinaemia and vascular disease. Recently Selhub et al. (23) analysed plasma homocysteine in 1160 elderly subjects. The mean plasma homocysteine concentration was increased in subjects within the lowest deciles of serum cobalamin and blood folate. These results suggest that marginal vitamin deficiencies are associated with hyperhomocysteinaemia. Their findings also indicated that elevated plasma homocysteine concentrations and suboptimal folate status were associated with an increased risk of carotid artery stenosis in elderly people. The observation (23) that the prevalence of carotid artery stenosis among the subjects in the lowest folate quartile remained significantly elevated even after adjustment for plasma homocysteine concentrations may indicate an independent effect of folate deficiency on atheroma formation and subsequent carotid artery stenosis. Recently, it was also demonstrated that inadequate folate intake was the main determinant of the homocysteine-related increase in carotid artery wall thickening (24). Furthermore, Pancharuniti et al. (25) have reported that low folate concentrations, but not serum cobalamin concentrations, are associated with coronary artery disease. The significant association between folate and vascular disease can probably be largely explained by its regulation of plasma homocysteine concentration.

In conclusion, the observation by Egerton et al. (18) and the findings of the present study indicate that the increased plasma homocysteine in the convalescent phase after an acute vascular event might be partly due to a lowering effect of plasma homocysteine at the time of the acute episode, and partly due to the fact that a marginal folate deficiency develops in many patients in the convalescent phase. It may therefore be of value for these patients to receive folate supplementation in the convalescent phase.

\section{Acknowledgements}

This work was supported by grants from the Medical Faculty, Lund University, the Swedish National Association Against Heart and Chest Disease and the Albert Påhlsson Foundation. 


\section{References}

1. Mudd SH, Harvey LL, Skovby F. Disorders of transsulfuration. In: Scriver CG, Beaudet AL, Sly WS, Valle D, editors. The metabolic basis of inherited disease. 6th ed. New York: McGraw-Hill Book Co, 1989:693-734.

2. Brattström L, Hardebo J, Hultberg B. Moderate homocysteinemia - a possible risk factor for arteriosclerotic cerebrovascular disease. Stroke 1984; 15:1012-6.

3. Boers GHJ, Smals AGH, Trijbels FJM, Fowler B, Bakkeren JAJM, Schoonderwaldt HC, et al. Heterozygosity for homocystinuria in premature peripheral and cerebral occlusive arterial disease. $\mathrm{N}$ Engl J Med 1985; 313:709-15.

4. Brattström $L$, Israelsson $B$, Norrving $B$, Bergqvist $D$, Thörne $\mathrm{J}$, Hultberg B, et al. Impaired homocysteine metabolism in early-onset cerebral and peripheral occlusive arterial disease effects of pyridoxine and folic acid treatment. Atherosclerosis $1990 ; 81: 51-60$.

5. Malinow MR, Kang SS, Taylor LM, Wong PWK, Coull B, Inahara $\mathrm{T}$, et al. Prevalence of hyperhomocysteinemia in patients with peripheral arterial occlusive disease. Circulation 1989; 79:1180-9.

6. Clarke R, Leslie D, Robinson K, Naughten E, Cahalane S, Fowler B, Graham I. Hyperhomocysteinemia: an indepent risk factor for vascular disease. N Engl J Med 1991; 324: 1149-55.

7. Malinow MR. Plasma homocysteine and arterial occlusive diseases: a mini-review. Clin Chem 1995; 41:173-6.

8. Lindenbaum J, Helaton EB, Savage DG, Brust JCM, Garrett TJ, Podell ER, et al. Neuropsychiatric disorders caused by cobalamin deficiency in the absence of anemia or macrocytosis. N Engl J Med 1988; 318: 1720-8.

9. Stabler SP, Marcell PD, Podell ER, Allen RH, Savage DG, Lindenbaum J. Elevation of total homocysteine in the serum of patients with cobalamin or folate deficiency detected by capillary gas chromatography-mass spectrometry. J Clin Invest 1988; 81:466-74.

10. Kang S-S, Wong PWK, Norusis M. Homocysteinemia due to folate deficiency. Metabolism 1987; 36:458-62.

11. Brattström L, Osraelsson B, Lindgärde F, Hultberg B. Higher total plasma homocysteine in vitamin B12 deficiency than in heterozygosity for homocystinuria due to cystathionine $\beta$-synthase deficiency. Metabolism 1988; 522-59.

12. Allen RH, Stabler SP, Savage DG, Lindenbaum J. Diagnosis of cobalamin deficiency I. Usefulness of serum methylmalonic acid and total homocysteine concentrations. Am J Hematol $1990 ; 34: 90-8$.

13. Lindenbaum J, Savage DG, Stabler SP, Allen RH. Diagnosis of cobalamin deficiency II. Relative sensitivities of serum cobalamin, methylmalonic acid and total homocysteine concentrations. Eur J Hemat 1990; 34:99-107.
14. Savage DG, Lindenbaum J, Stabler SP, Allen RH. Sensitivity of serum methylmalonic acid and total homocysteine determinations for diagnosing cobalamin and folate deficiencies. Am J Med 1994; 96:239-46.

15. Ubbink JB. Vitamin nutrition status and homocysteine: an atherogenic risk factor. Nutr Rev 1994; 52:383-93.

16. Lindgren A, Brattström L, Norrving B, Hultberg B, Andersson A, Johansson B. Plasma homocysteine in the acute and convalescent phases after stroke. Stroke 1995; 26:795-800.

17. Landgren F, Israelsson B, Lindgren A, Hultberg B, Andersson A, Brattström L. Plasma homocysteine in acute myocardial infarction: homocysteine-lowering effect of folic acid. J Int Med 1995; 237:381-8.

18. Egerton W, Silberberg J, Crooks R, Ray C, Xie L, Dudman N. Serial measures of plasma homocysteine after acute myocardial infarction. Am J Cardiol 1996; 77:759-61.

19. Andersson A, Brattström B, Israelsson B, Isaksson A, Hamfelt A, Hultberg B. Plasma homocysteine before and after methionine loading with regard to age, gender and menopausal status. Eur J Clin Invest 1992; 22:78-87.

20. Brattström L, Lindgren A, Israelsson B, Andersson A, Hultberg $\mathrm{B}$. Homocysteine and cysteine: determinants of plasma levels in middle-aged and elderly subjects. J Intern Med 1994; 236:633-41.

21. Andersson A, Isaksson A, Brattström L, Hultberg B. Homocysteine and other thiols determined in plasma by HPLC and thiol-specific postcolumn derivatization. Clin Chem 1993; 39:1590-7.

22. Davis RE, Nicol DJ. Folic acid. Int J Biochem 1988; 20:133-9.

23. Selhub J, Jacques PF, Wilson PWF, Rush D, Rosenberg IH. Vitamin status and intake as primary determinants of homocysteinemia in an elderly population. J Am Med Ass 1993; 270:2693-8.

24. Selhub J, Jacques PF, Bostom AG. Association between plasma homocysteine concentrations and extracranial carotidartery stenosis. New Engl J Med 1995; 332:286-91.

25. Pancharuniti N, Lewis CA, Sauberlich HE, Perkins LL, Go RCP, Alvarez JO, et al. Plasma homocysteine, folate and vitamin B-12 concentrations and risk for early-onset coronary artery disease. Am J Clin Nutr 1994; 59:940-8.

Received June 25/October 7, 1996

Corresponding author: B. Hultberg, PhD, MD, Department of Clinical Chemistry, University Hospital, S-22185 Lund, Sweden 